\title{
Cuerpo extraño alojado en el tercer ventrículo por arma neumática sin repercusiones neurológicas, a 12 años de seguimiento. Reporte de un caso
}

\section{Foreing body lodged in third ventricle, by pneumatic weapon without neurological damage to 12 year follow up. Case report}

Alejandro Barrón-Balderas, ${ }^{1}$ Mireya Robledo-Aceves, ${ }^{1}$ Ramiro López-Elizalde, ${ }^{2}$ Javier Álvaro Barriga-Marín ${ }^{1}$

Resumen

ANTECEDENTES: Las lesiones por proyectil de arma neumática pueden ser mortales o con múltiples secuelas, principalmente si afectan el sistema nervioso central.

CASO CLínICO: Paciente de 8 años, que sufrió un impacto de proyectil neumático (rifle de balines perdigón de $5.5 \mathrm{~mm}$ de calibre), a una distancia aproximada de 15 $\mathrm{cm}$ del rostro. No refirió pérdida del conocimiento, convulsiones ni vómito, solo dolor y sangrado abundante en el sitio de entrada del objeto. A su ingreso al hospital se encontraron constantes fisiológicas normales. En la región frontal se observó una herida circular, con bordes irregulares de $6 \mathrm{~mm}$. Las radiografías de cráneo evidenciaron un cuerpo extraño radiopaco alojado en la parte central del interior del cráneo. La tomografía simple de cráneo reportó un trayecto de entrada en línea paramediana izquierda, además de ruptura del ventrículo lateral izquierdo y alojamiento del cuerpo extraño en el tercer ventrículo. El equipo de Neurocirugía descartó llevar a cabo algún procedimiento quirúrgico, debido a la localización del cuerpo extraño. El paciente fue hospitalizado durante 10 días, recibió tratamiento con ceftriaxona $(100 \mathrm{mg} / \mathrm{kg} /$ día) por cinco días. Permaneció asintomático, con cicatrización adecuada del orificio de entrada. En la última revisión, 12 años después del evento, la exploración física y neurológica no reportó manifestaciones ni complicaciones. Actualmente tiene 20 años y mantiene un buen estado de salud.

CONCLUSIONES: Es necesario que las "armas de juguete" sean etiquetadas como artículos peligrosos. Se recomienda adecuar leyes para la venta y uso de las armas neumáticas. PALABRAS CLAVE: Armas neumáticas; balines perdigón; tercer ventrículo; cuerpo extraño; ceftriaxona.

Abstract

BACKGROUND: The projectile injuries of pneumatic weapons, due to their mechanics, can produce fatal injuries or with multiple sequels, mainly of the central nervous system. CLINICAL CASE: 8-year-old patient, who suffered an impact from a pneumatic projectile (5.5 $\mathrm{mm}$ caliber rifle), at a distance of approximately $15 \mathrm{~cm}$ from the face. He reported no loss of consciousness, seizures or vomiting, only pain and heavy bleeding at the site of entry of the object. Upon admission to the hospital, normal physiological constants were found. In the frontal region a circular wound was observed, with irregular edges of $6 \mathrm{~mm}$. Skull x-rays showed a radiopaque foreign body lodged in the central part of the inside of the skull. Simple skull tomography reported an entry path in the left paramedian line, in addition to a rupture of the left lateral ventricle and accommodation of the foreign body in the third ventricle. The Neurosurgery team ruled out performing a surgical procedure, due to the location of the foreign body. The patient was hospitalized for 10 days, received treatment with ceftriaxone $(100 \mathrm{mg} / \mathrm{kg} /$ day $)$ for five days. He remained asymptomatic, with adequate healing of the entrance hole. In the last review, 12 years after the event, the physical and neurological examination did
${ }^{1}$ Servicio de Pediatría.
${ }^{2}$ Servicio de Neurocirugía.

Hospital Civil de Guadalajara Dr. Juan I. Menchaca, Guadalajara, Jalisco, México.

Recibido: 29 de octubre 2018

Aceptado: 22 de agosto 2019

Correspondencia

Mireya Robledo-Aceves

myreace@yahoo.es

Este artículo debe citarse como Barrón-Balderas A, Robledo-Aceves M, López-Elizalde R, Barriga-Marín JA. Cuerpo extraño alojado en el tercer ventrículo por arma neumática sin repercusiones neurológicas, a 12 años de seguimiento. Reporte de un caso. Acta Pediatr Mex. 2019;40(6):335-41. 
not report manifestations or complications. He is currently 20 years old and maintains a good state of health.

CONCLUSIONS: It is necessary that "toy weapons" be labeled as dangerous items. It is recommended to adapt laws for the sale and use of pneumatic weapons.

KEYWORDS: Pneumatic weapons; Bullet ballon; Third ventricle; Foreign bodies; Ceftriaxone.

\section{ANTECEDENTES}

En 2016 se reportaron 251,000 defunciones asociadas con armas de fuego en Brasil, Estados Unidos, México, Colombia, Venezuela y Guatemala. El $64 \%$ se relacionó con homicidios, $27 \%$ con suicidios y $9 \%$ por arma de fuego no intencional. ${ }^{1}$

En México, el artículo décimo de la Constitución Mexicana dicta: "Ios habitantes de los Estados Unidos Mexicanos tienen derecho a poseer armas en su domicilio, para su seguridad y legítima defensa, con excepción de las prohibidas por la Ley Federal y de las reservadas para el uso exclusivo del Ejército, Armada, Fuerza Aérea y Guardia Nacional". Sin embargo, con el argumento de proteger su hogar e integridad física, debido a la elevada tasa de inseguridad que existe en la actualidad, ha facilitado el comercio de armas de fuego y sus similares de manera exponencial.

Las armas neumáticas (rifles o pistolas de aire) se utilizan en todo el mundo como instrumentos de diversión, juguetes en parques de atracciones, entrenamiento preliminar con armas de fuego y cacería de aves. ${ }^{2}$ En México, la Secretaría de la Defensa Nacional (SEDENA), con base en la Ley Federal de Armas de Fuego y Explosivos, no considera estos instrumentos en el ámbito legal de la regulación de armas, ni existe restricción en la edad del usuario, tal como sucede en otros países; por tanto, permite a una persona acceder con bastante facilidad a este tipo de objetos. ${ }^{2,3}$
Según lo reportado por Kumar, cada año se comercializan 3.2 millones de armas neumáticas en Estados Unidos, y 1 de cada 3 niños posee una de éstas. ${ }^{4}$ Las armas neumáticas son propiedad común en infantes, ya sea obtenidas por su bajo costo o como regalo, sin ser reconocidos los peligros que conlleva un instrumento de este tipo. En el ámbito pediátrico, la bibliografía reporta que la mayor parte de las lesiones intracraneales con arma de aire comprimido ocurre de manera accidental. ${ }^{4}$

En las últimas décadas se han incrementado los accidentes y la violencia relacionados con armas como agente causal, y con ello las heridas provocadas por este tipo de instrumentos; desafortunadamente, la población pediátrica no se encuentra exenta de este riesgo. ${ }^{5}$

En México existen pocas publicaciones relacionados con este tipo de percances. Las heridas por arma de proyección balística en niños son una causa significativa de morbilidad y mortalidad en la actualidad. ${ }^{5}$

Los pacientes pediátricos son más susceptibles de sufrir lesiones intracraneales que el resto de la población, en especial a menor edad del paciente. Esta susceptibilidad se debe a la superficie craneal proporcionalmente mayor, musculatura relativamente débil, plano óseo más fino y deformable, y mayor contenido de agua y disminuido de mielina, comparados con el paciente adulto. ${ }^{6}$ Las heridas de entrada se reportan con mayor frecuencia en las regiones frontal y temporal. ${ }^{7}$ 
Giudice $^{7}$ define las armas de proyección balística como "instrumentos mecánicos, automáticos o semiautomáticos, capaces de disparar un proyectil al aire." Para que un disparo se lleve a efecto, el proyectil debe ir precedido de una energía cinética que le confiera capacidad de desplazamiento mediante una fuerza propulsora. ${ }^{8}$ Dicha fuerza puede variar dependiendo su origen, es decir, armas de fuego, neumáticas, fluidos y proyección luminosas. Las armas neumáticas, a diferencia de las de fuego, utilizan aire comprimido en lugar de pólvora para impulsar un proyectil. ${ }^{9}$

Se reconocen diversos tipos de proyectiles para las armas neumáticas; se clasifican según su forma y los más comunes son los diábolos (pointed pellet) y los balines perdigón (bullet ballon). ${ }^{10}$ Los proyectiles pueden variar dependiendo el calibre: para las armas que utilizan diábolos existen calibres de 4.5 (0.177 pulgadas oficial para competencia), 6.35 (0.25 pulgadas) y $5 \mathrm{~mm}$ ( 0.20 pulgadas). Los balines perdigón cuentan calibres de 4.4 (0.17 pulgadas) y 5.5 $\mathrm{mm}(0.22$ pulgadas), consistentes en esferas de acero, frecuentemente utilizados en pistolas y rifles, por su facilidad para el funcionamiento semiautomático. ${ }^{7,9,10}$ Las pistolas y los rifles de aire comprimido son capaces de alcanzar una velocidad de impacto de 83 a 106 y 274 m/seg, respectivamente. ${ }^{11-14}$

\section{CASO CLÍNICO}

Niño de 8 años, residente de una población rural localizada en el municipio de Venustiano Carranza, Michoacán, México, que se encontraba en una zona agreste, de cacería, con un rifle neumático de "balines", quien al dispararlo se trabó el mecanismo percutor, por lo que decidió revisar el cañón a una distancia aproximada de $15 \mathrm{~cm}$ del rostro, produciéndose el disparo y la expulsión de manera espontánea de un balín de $5.5 \mathrm{~mm}$ de calibre.
Desde ese momento se refirió sin pérdida del conocimiento, convulsiones ni vómito, solo dolor y sangrado abundante en el sitio de entrada, motivo por el que fue llevado al servicio de Urgencias del Hospital Civil de Guadalajara Dr. Juan I. Menchaca. A su ingreso se encontraron: frecuencia cardiaca de $81 \mathrm{lpm}$, frecuencia respiratoria de $24 \mathrm{rpm}$, temperatura $37^{\circ} \mathrm{C}$, tensión arterial 100/65 mmHg. El paciente se percibía consciente, orientado con 15 puntos en la Escala de coma de Glasgow; dolor local leve en el sitio de entrada del proyectil, con respuesta cognitiva adecuada, sin afectación de los pares craneales ni daño neurológico adicional; las extremidades tenían movilidad y fuerza adecuadas, reflejos osteotendinosos normales.

En la región frontal se observó una herida circular, con bordes irregulares de aproximadamente $6 \mathrm{~mm}$ de diámetro. Los exámenes de laboratorio reportaron: hemoglobina de $12 \mathrm{~g} / \mathrm{dL}$, hematocrito $34.4 \%$, plaquetas $270 \times 103 / \mathrm{mm}^{3}$, leucocitos $14.5 \times 103 / \mathrm{mm}^{3}$, tiempo de protrombina 14.4 seg y tiempo de tromboplastina parcial activada $23.5 \mathrm{seg}$.

Las radiografías de cráneo (Figura 1) evidenciaron un cuerpo extraño radiopaco alojado en la parte central del interior del cráneo. La tomografía simple de cráneo (Figura 2) reportó un trayecto de entrada en línea paramediana izquierda, además de ruptura del ventrículo lateral izquierdo y alojamiento del cuerpo extraño en el tercer ventrículo. El servicio de Neurología y Neurocirugía realizó las pruebas de habilidades motoras y sensoriales, incluyendo los pares craneales, audición, habla, agudeza visual, coordinación, equilibrio, estado mental, además de cambios en el estado de ánimo y conducta durante su hospitalización, que resultaron normales en todo momento.

En la evaluación psicológica, desde su llegada se mostró sin rechazo a la figura de autoridad, coo- 


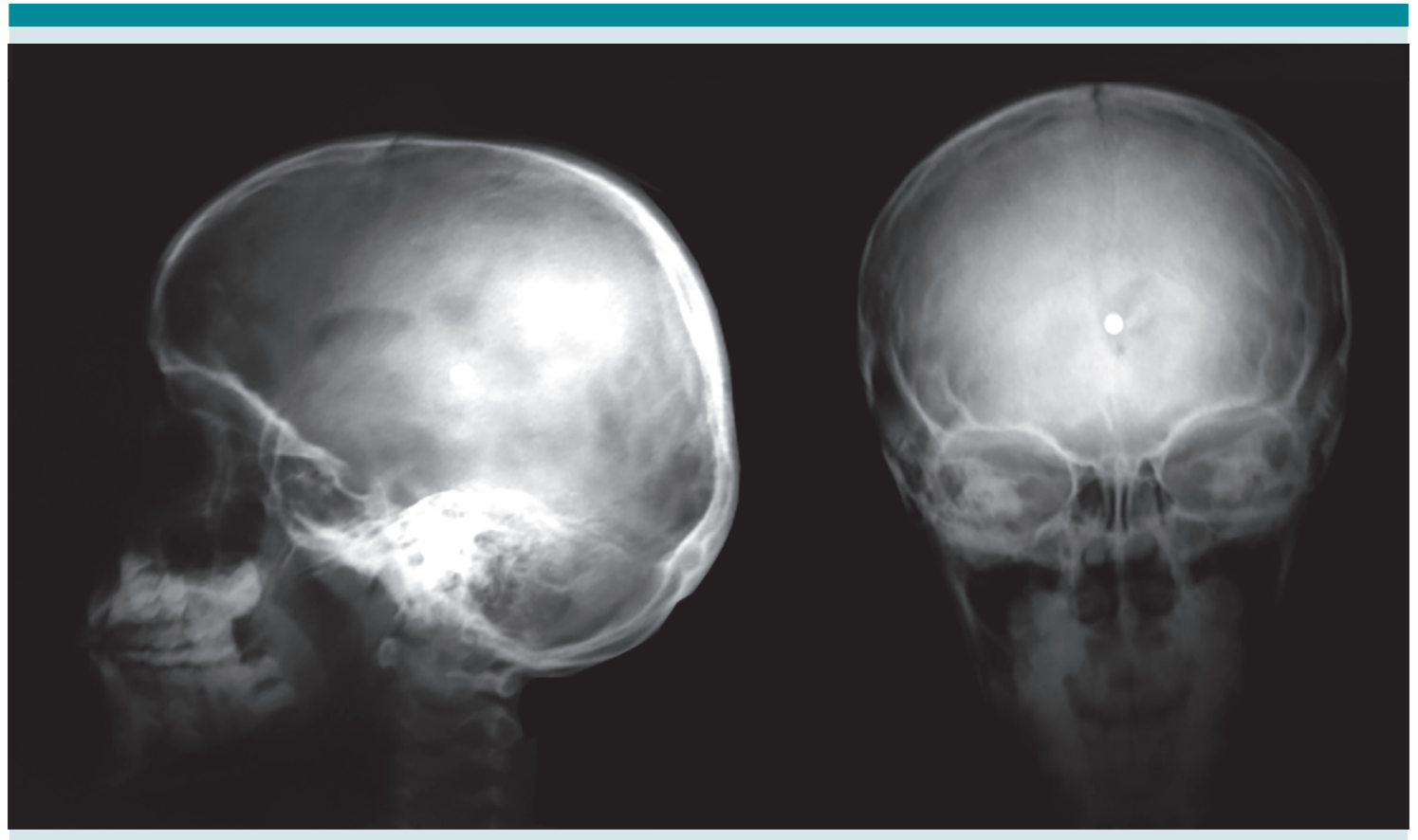

Figura 1. Radiografía AP y lateral de cráneo, donde se observa un objeto radiopaco.

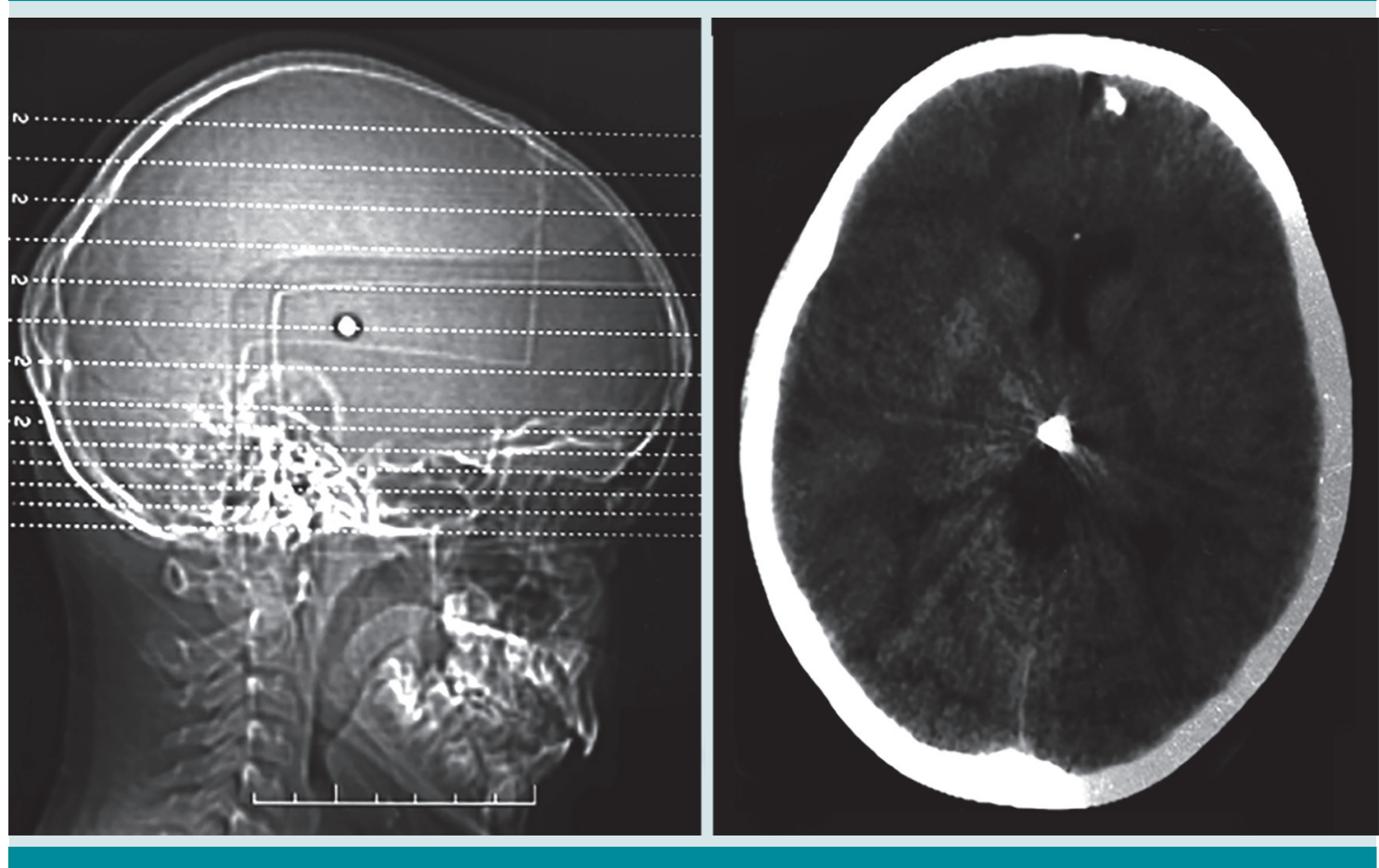

Figura 2. Tomografía simple de cráneo, con cuerpo radiopaco en el tercer ventrículo. 
perador, coherente, con lenguaje fluido, acorde con la edad cronológica, manteniendo contacto visual con el entrevistador, coherencia con la expresión de emociones y discurso planteado por el menor. No se percibieron movimientos estereotipados que denotaran ansiedad ni estrés; por tanto, no fue necesaria la intervención de primeros auxilios psicológicos.

El equipo de Neurocirugía descartó llevar a cabo algún procedimiento quirúrgico, debido a la localización del cuerpo extraño. El paciente fue hospitalizado durante 10 días, recibió tratamiento con ceftriaxona $(100 \mathrm{mg} / \mathrm{kg} / \mathrm{día})$ por cinco días. Permaneció asintomático, con cicatrización adecuada del orificio de entrada.

Continuó en seguimiento en la consulta externa después de 1 mes del accidente y posteriormente cada cuatro años. En la última revisión, 12 años después del evento, la exploración física y neurológica no reportó manifestaciones ni complicaciones. Actualmente tiene 20 años, está casado, es padre de un hijo y se dedica al comercio y la albañilería.

\section{DISCUSIÓN}

En las últimas décadas se ha incrementado la tasa de accidente y violencia relacionada con armas como agente causal. Desafortunadamente la población pediátrica no se encuentra exenta de este riesgo.

Los niños, a diferencia de los adultos, tienen mayor riesgo de lesión intracraneal, debido a los diferentes grados de desarrollo de los tejidos, además del grosor del cráneo, lo que condiciona la penetración de objetos a velocidades bajas.

En 2016, Kumar ${ }^{4}$ reportó 17 pacientes lesionados por arma neumática de tres diferentes hospitales de Estados Unidos en 17 años; el promedio de edad era de 8 años; $86 \%$ correspondió al género masculino, en $71 \%$ fue consecuencia de accidente y $7 \%$ vinculado con violencia por robo; no se reportó tentativa suicida. El 57\% tuvo un orifico de entrada en la frente; las lesiones reportadas se consideraron graves: hemorragia subdural, fractura de cráneo, edema cerebral, hemorragia intraparenquimatosa, hematoma subdural, hemorragia interventricular y pseudoaneurisma. El 71\% de los pacientes requirió tratamiento quirúrgico y $43 \%$ tuvo secuelas neurológicas permanentes (epilepsia, deficiencias cognitivas, hidrocefalia, diplopía, disminución de campos visuales y en algunos casos ceguera); sin embargo, no se reportaron defunciones.

Lo trascendente de nuestro caso fue la evolución benevolente y sin alteraciones neurológicas a corto y mediano plazo, resultado de las siguientes condiciones:

a. La baja velocidad del proyectil, calculada entre 122 a $274 \mathrm{~m} / \mathrm{seg}$

b. La distancia existente entre el cañón y el cráneo

c. El cuerpo hueco del balín

d. La permanencia lineal en la dirección que tomó el obús respecto al punto anatómico de entrada y el sitio final de alojamiento, cruzando la línea media interhemisférica (Figura 3)

Estas condiciones permitieron que no se deformara ni fracturara el obús, evitando con ello la formación de esquirlas que se convertieran en múltiples fragmentos lesivos, disminuyendo la posibilidad de secuelas e incrementando la supervivencia. ${ }^{13-17}$

La velocidad alcanzada por el balín permitió la fricción necesaria para generar el calor suficiente y disminuir el riesgo de contaminación e inoculación de patógenos en el sitio final de alojamiento. 


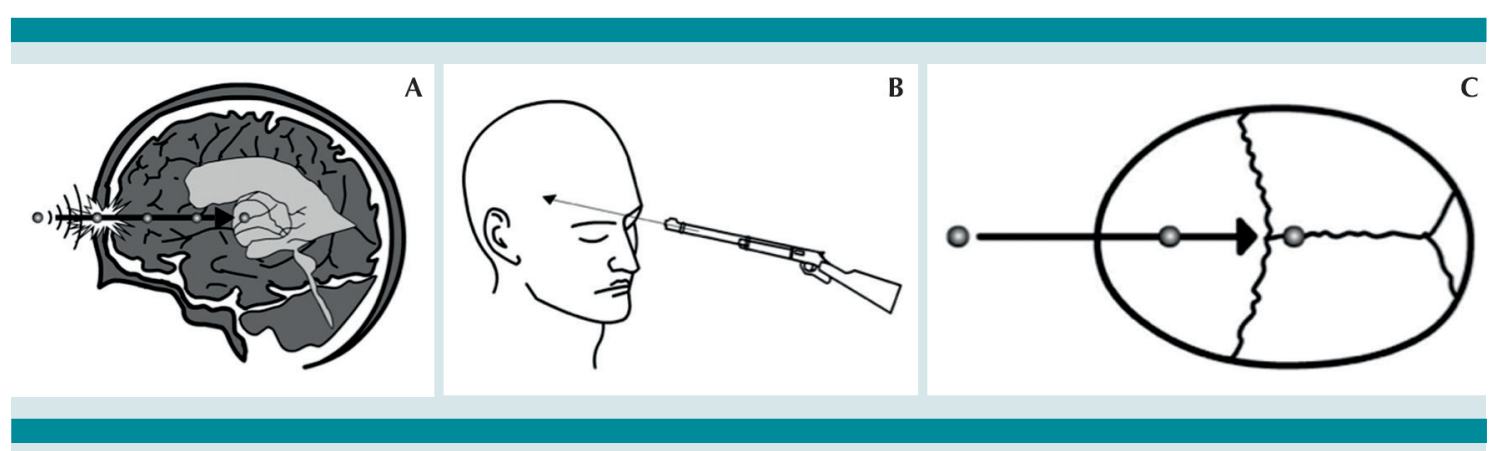

Figura 3. Representación de la trayectoria del proyectil. A) Entrada hasta su alojamiento en el tercer ventrículo, B y C) trayectoria del proyectil cruzando la línea media interhemisférica.

Coincidimos con la publicación de Kumar, ${ }^{4}$ quien reporta que la edad promedio de los pacientes es de 8 años, con predominio del sexo masculino las lesiones suelen resultar de un accidente, así como la herida de entrada predomina en la región frontal. Sin embargo, encontramos diferencia de lo reportado respecto a la evolución, pues en nuestro paciente fue buena, sin repercusiones a corto y largo plazo, y su pronóstico y calidad de vida fue bueno. Esto sugiere una suma de acontecimientos afortunados acorde con los mecanismos de la cinética de la balística.

El bajo costo de las armas neumáticas, la falta de supervisión por parte de un adulto y la ausencia de un adiestrador formal en el manejo y cuidado de estas armas, por ser consideradas juguetes o equipos deportivos, además del uso y costumbre en nuestra población, aunados a la deficiente legislación al respecto y principalmente la orientación educativa a propósito del uso de este tipo de armas "consideradas poco peligrosas", son los principales factores que contribuyen a la incidencia de accidentes por armas de aire comprimido, facilitando que los niños sufran lesiones con secuelas que pueden ser permanentes.

\section{CONCLUSIÓN}

Las armas neumáticas son artefactos peligrosos que se comercializan como juguetes para los niños. Debido al avance tecnológico en éstas, pueden generar velocidades de disparo capaces de atravesar el tejido óseo. Los niños, en particular, son vulnerables a las lesiones por armas neumáticas, pues su cráneo se encuentra en pleno desarrollo, aunado a la falta de conciencia del peligro que suponen y lo atractivo que resulta el comercio de estas armas para los pequeños. Es necesario que las "armas de juguete" sean etiquetadas como artículos peligrosos; por lo tanto, se recomienda adecuar leyes para la venta y uso de las armas neumáticas, recalcando que la velocidad de impacto sea baja, con niveles que no permitan la penetración de la piel y, sobre todo, del tejido óseo.

\section{REFERENCIAS}

1. The Global Burden of Disease 2016 Injury Collaborators. Global Mortality From Firearms, 1990-2016. JAMA 2018;320(8):792-814. doi:10.1001/jama.2018.10060

2. Kuligod FS, et al. Air gun - A deadly toy?: A case report. Med Sci Law 2005;45(4):1-4. DOI: 10.1258/rsmmsl.46.2.177

3. Fuentes-Mallozzi DA. Herida por proyectil de arma neumática. An Orl Mex 2008;53(4):182-5. https://www.medigraphic.com/cgi-bin/new/resumen.cgi?IDARTICULO=27724 
Barrón-Balderas A, et al. Cuerpo extraño en el tercer ventrículo

4. Kumar $\mathrm{R}$, et al. Penetrating head injuries in children due to $B B$ and pellet guns: a poorly recognized public health risk. J Neurosurg Pediatr 2016;17(2):215-221. DOI: 10.3171/2015.6.PEDS15148

5. García-Pinto G,. Heridas por arma de fuego en la población pediátrica. Rev Sanid Milit Mex 2012;66(3):105-109. http:// nietoeditores.com.mx/nieto/Sanidad\%20Militar/2015/ may-jun/epidemiologia_heridas.pdf

6. Asociación Española de Pediatría, Sociedad Española de Urgencias Pediátricas. (2010). Protocolos diagnosticoterapéuticos de Urgencias Pediátricas SEUP-AEP (2aed.). Recuperado de https://www.aeped.es/documentos/ protocolos-urgencias-pediatricas-en-revision

7. Giudice M. La Criminalística, la lógica y la prueba en el COPP. La nueva forma de concebir Is pruebas. 7a ed. Caracas: Editorial Vadell Hermanos editores, 2014;8-200.

8. Baeza-Herrera C, Baeza-Herrera MA. Heridas provocadas por proyectil en el área de Pediatría. Gac Méd Méx 1998;134(3):289-95. http://www.anmm.org.mx/ bgmm/1864_2007/1998-134-3-289-295.pdf

9. Di Maio VJM. Gunshot wound. Practical aspects of firearms, ballistics, and forensic techniques. $2^{\text {nd }}$ ed. New York: CRC Press LLC; 1999, 227-30.
10. Uquillas-Sota H. Pólvora y perdigones. $1^{\text {ra }}$ ed. Morelos: Impresores de Morelos, 1982;16-98.

11. Morgan JC, et al. Air gun injuries of the abdomen in children. Arch Surg. 1984;119:14-18. DOI: 10.1001/archsurg.1984.01390240073013

12. Greensher J, et al. Injuries related to "Toy" firearms. Pediatr 1987;79(3):473-74. https://www.cdc.gov/mmwr/preview/ mmwrhtml/00039773.htm

13. Sánchez-Vázquez MA, et añ. Herida incidental por proyectil de arma de fuego en un niño. Arch Neurocien 2001;6(3):153-158.

14. Comité internacional de la Cruz Roja. Balística de las heridas, Ginebra Suiza: 2008. p.10-35

15. Laraque D. Injury risk of nonpowder guns. Pediatr 2004;114(5):1357-61. DOI: 10.1542/peds.2004-1799

16. Fonseca-Lainez $\mathrm{M}$, et al. Heridas por perdigones de cuello y torso, el nuevo desafío. Rev Med Post 2002;7(1):79-83. http://www.bvs.hn/RMP/pdf/2002/pdf/Vol7-1-2002-16. pdf

17. Net Press. Investigación criminal. Balística Forense-Daños producidos. Consultado 10 Enero 2019 Sitio web: http:// netpress1.blogspot.mx/2007/ 